INPLASY

PROTOCOL

To cite: Luo et al. Evaluation of tranexamic acid after total hip arthroplasty over 60 years old in China: a Systematic Review and Meta-analysis. Inplasy protocol 202210048. doi: 10.37766/inplasy2022.1.0048

Received: 10 January 2022

Published: 10 January 2022

Corresponding author:

Peng Xu

sousou369@163.com

Author Affiliation:

Xi'an jiaotong university.

Support: None.

Review Stage at time of this submission: Data analysis Completed but not published.

Conflicts of interest:

None declared.

\section{Evaluation of tranexamic acid after total hip arthroplasty over 60 years old in China: a Systematic Review and Meta-analysis}

Luo, P1; Gao, Fq²; Zhang, Nf3; Yu, Hw; Xu, K5; Xu, P6.

Review question / Objective: The purpose of this metaanalysis was to evaluate the efficacy of tranexamic acid after total hip arthroplasty in patients older than 60 years old in China by meta-analysis.

Participant or population: All trials included in our study meet the following criteria: (1) All studies were original RCTs; (2) The mean age of patients for each study was $\geq 60$ years old; (3) Patients were received total hip arthroplasty in all studies; (4) All studies included oral and iv or topical groups, with a comparison of outcomes between the two groups; (5) The full text of the included literature can be obtained, and the measurement data of hemoglobin drop, total blood loss, transfusion rate, complication, length of stay can be extracted. The following studies were excluded from the meta-analysis: nonrandomized studies; the patients with age<60; studies not suitable with the inclusive criteria; and articles for which we were unable to obtain the full text and relevant data for pooled analysis.

INPLASY registration number: This protocol was registered with the International Platform of Registered Systematic Review and Meta-Analysis Protocols (INPLASY) on 10 January 2022 and was last updated on 10 January 2022 (registration number INPLASY202210048).

\section{INTRODUCTION}

Review question / Objective: The purpose of this meta-analysis was to evaluate the efficacy of tranexamic acid after total hip arthroplasty in patients older than 60 years old in China by meta-analysis.
Condition being studied: With the development of medical technology, total hip arthroplasty (THA) has become one of the most satisfactory operations in orthopedic treatment, especially for patients with limited movement and pain of the hip joint. THA can relieve pain and restore hip function, significantly improving 
the quality of life of patients after operation. However, THA usually causes significant blood loss, especially in the first week after surgery, and leads to anemia, which puts patients at higher risk of cardiopulmonary events, longer recovery periods and higher medical costs. With the increase of age, the blood viscosity of the human body increases, and it is more likely to suffer from high blood pressure and arteriosclerosis and thrombus. Because most THA operations are performed on the elderly. Therefore, the risk of postoperative thromboembolic complications in the elderly is increased. Tranexamic acid is a kind of synthetic amino acid, which can effectively reduce blood loss and adverse outcome after THA by competitively inhibiting the activation of plasminogen and thus inhibiting the function of degrading blood clots. A number of studies have shown that local use of tranexamic acid (TXA) can achieve similar or even better hemostatic efficacy than intravenous use of TXA And multiple oral TXA has the best hemostatic effect compared with a single oral administration. However, the efficacy and safety of oral vs. intravenous and local TXA administration are still controversial. Some authors suggest that local administration of TXA can reduce blood loss and transfusion rates, but others believe that intravenous administration is the way to achieve the maximum effect. However, some authors believe that the efficacy of oral administration, intravenous administration and local administration is similar, but which mode of administration is more safe and effective is still controversial, so the main purpose of this article is to evaluate the efficacy of tranexamic acid after total hip arthroplasty in patients older than 60 years old by metaanalysis.

\section{METHODS}

Search strategy: In order to obtain all the literature related to our research, in the first step, two reviewers independently used the keywords combined with free words to search English-based electronic databases according to Cochrane Collaboration guidelines, such as PubMed
(2017 to September 1, 2020), Embase (2017 to September 1, 2020), ScienceDirect (2017 to September 1, 2020), and Cochrane library (2017 to September 1, 2020). In the second step, the potentially related literature was searched from the list of references of all included studies. We used Medical Subject Headings (MeSH) terms and corresponding keywords to search the following terms "total hip replacement or total hip arthroplasty or THA", "Tranexamic acid or Tranexamic or TXA", with the Boolean operators "AND or OR". Two researchers independently conducted preliminary screening by reading the titles and abstracts of the retrieved literature. Then, the selected literature should be further filtered by reading the full text as much as possible. All disagreeable literature was resolved after discussion.

Participant or population: All trials included in our study meet the following criteria: (1) All studies were original RCTs; (2) The mean age of patients for each study was $\geq$ 60 years old; (3) Patients were received total hip arthroplasty in all studies; (4) All studies included oral and iv or topical groups, with a comparison of outcomes between the two groups; (5) The full text of the included literature can be obtained, and the measurement data of hemoglobin drop, total blood loss, transfusion rate, complication, length of stay can be extracted. The following studies were excluded from the meta-analysis: nonrandomized studies; the patients with age $<60$; studies not suitable with the inclusive criteria; and articles for which we were unable to obtain the full text and relevant data for pooled analysis.

Intervention: Tranexamic acid.

Comparator: We analyzed continuous data using weighted mean differences (WMD) and their $95 \%$ confidence interval $(\mathrm{Cl})$, such as hemoglobin drop, total blood loss and length of stay. And we used risk ratio (RR) with $95 \%$ Cls to weigh the effect interval of discontinuous outcomes.

Study designs to be included: This metaanalysis included a total of 6 RCTs 
published between 2017 and 2020 . Characteristics of all the studies included in the meta-analysis are shown in Table 1. All studies compared differences in the therapeutic effects of TXA in Patients aged 60 or older who underwent total hip replacement.

Eligibility criteria: In the first step, we searched multiple databases and identified 46 records. After removing the duplicate records and the irrelevant records by reading the titles and abstracts, a total of 9 records were selected and the next step was to read the full text. According to the inclusion criteria, records of non-RCT, records with an average age $<60$ years old, and records for which data could not be extracted were excluded.

\section{Information sources: Pubmed.}

Main outcome(s): The main outcome index was hemoglobin loss.

Additional outcome(s): The second outcome index was total blood loss, blood transfusion rate, hospital stay and complications. And each outcome index was analyzed by subgroup analysis according to the mode of medication and the number of oral administration.

Data management: The meta-analysis was performed using RevMan $\mathbf{5 . 3}$ for Windows (Cochrane Collaboration, Oxford, UK).

Quality assessment / Risk of bias analysis: The risk of bias in each included RCT was assessed according to the Cochrane Handbook for Systematic Reviews. The evaluation of bias can be divided into 7 sections: random sequence generation, allocation concealment, blinding of participant and personnel, blinding of outcome assessment, incomplete outcome data, selective reporting, and other bias. Each section can have a high risk of bias, low risk of bias and unclear risk of bias depending on the actual content of the included study.

Strategy of data synthesis: Merge data according to outcome metrics.
Subgroup analysis: Each outcome index was analyzed by subgroup analysis according to the mode of medication and the number of oral administration.

Sensitivity analysis: Statistical heterogeneity was calculated by using a chi-square test and 12 test. It is considered that the 12 values of $25 \%, 50 \%$, and $75 \%$ indicate low, moderate and high heterogeneity, respectively. When $12 \leq 50 \%$, we performed a fixed-effect model for the meta-analysis.

Language: This article only contains systematic research in English.

Country(ies) involved: China.

Keywords: tranexamic acid, total hip arthroplasty, meta-analysis, oral.

Contributions of each author:

Author 1 - Pan Luo - Author 1 drafted the manuscript.

Email: 15610691516@163.com

Author 2 - Fuqiang Gao - The author provided statistical expertise.

Email: gaofuqiang@bjmu.edu.cn

Author 3 - Nianfei Zhang - The author contributed to the development of the selection criteria, and the risk of bias assessment strategy.

Email: zhangnianfei@hotmail.com

Author 4 - Hongwei Yu - The author read, provided feedback and approved the final manuscript.

Email: yuhw416@163.com

Author 5 - Ke Xu - The author read, provided feedback and approved the final manuscript.

Email: santxuke1986@126.com

Author 6 - Peng Xu - The author read, provided feedback and approved the final manuscript.

Email: sousou369@163.com 